\title{
SOME NEW INTEGRAL INEQUALITIES
}

\author{
LI YIN, DA-WEI NIU AND FENG QI
}

Abstract. In the paper, some new integral inequalities are presented by using analytic methods.

\section{Introduction}

In [31] and its preprint [30], the following problem was posed by the third author.

Open Problem 1.1. Under what conditions does the inequality

$$
\int_{a}^{b}[f(x)]^{p} \mathrm{~d} x \geq\left[\int_{a}^{b} f(x) \mathrm{d} x\right]^{p-1}
$$

hold for $p>1$ ?

Since then, this problem has been stimulating much interest of many mathematicians. In recent years, the third author has collected over forty articles devoted to answering and generalizing this open problem and to applying inequalities of this type. For potential availability to interested readers, we list the collection in the list of references of this paper.

In [33] and its preprint [48], the following result was obtained.

Theorem 1.1 ([33]). Let $f$ be a continuous function on $[a, b]$. If

$$
\int_{a}^{b} f(x) \mathrm{d} x \geq(b-a)^{p-1}
$$

for some $p>1$, then the inequality (1.1) is true.

Later in [32, p. 4, Theorem 1.1], an alternative condition for the inequality (1.1) to be valid was procured.

Corresponding author: Feng Qi.

2010 Mathematics Subject Classification. 26D15.

Key words and phrases. Integral inequality, Cauchy's mean value theorem, Minkowski's inequality, Hölder's inequality, induction.

The first author was supported partially by the Science Foundation of Binzhou University under grant BZXYL1303. 
Theorem 1.2 ([32]). Let $f(x)$ be continuous and not identically zero on $[a, b]$ and differentiable on $(a, b)$ with $f(a)=0$, and let $\alpha, \beta$ be positive real numbers such that $\alpha>\beta>1$. If

$$
\left[f^{(\alpha-\beta) /(\beta-1)}(x)\right]^{\prime} \gtreqless \frac{(\alpha-\beta) \beta^{1 /(\beta-1)}}{\alpha-1}
$$

for all $x \in(a, b)$, then

$$
\int_{a}^{b}[f(x)]^{\alpha} \mathrm{d} x \gtreqless\left[\int_{a}^{b} f(x) \mathrm{d} x\right]^{\beta} .
$$

In [3, p. 124, Theorem C], a different form of the inequality (1.1) was established, which can be reformulated as Theorem 1.3 below.

Theorem 1.3 ([3]). If $f(x)$ is a continuous function on $[a, b]$ such that $f(a) \geq 0$ and $f^{\prime}(x) \geq p \geq$ 1 on $(a, b)$, then

$$
\int_{a}^{b}[f(x)]^{p+2} \mathrm{~d} x \geq \frac{1}{(b-a)^{p-1}}\left[\int_{a}^{b} f(x) \mathrm{d} x\right]^{p+1} .
$$

The main purpose of this paper is to generalize the above-mentioned results and to present some new integral inequalities.

\section{Lemmas}

For generalizing Theorems 1.1 to 1.3 , we need the following lemmas.

Lemma 2.1. Let $x_{1}, x_{2}, \ldots, x_{n}$ and $y_{1}, y_{2}, \ldots, y_{n}$ be positive numbers. For $p, q>1$ with $\frac{1}{p}+\frac{1}{q}=1$, we have

$$
\sum_{k=1}^{n} \frac{x_{k}^{p}}{y_{k}^{p / q}} \geq \frac{\left(\sum_{k=1}^{n} x_{k}\right)^{p}}{\left(\sum_{k=1}^{n} y_{k}\right)^{p / q}} .
$$

The equality in (2.1) holds if and only if $\frac{x_{1}}{y_{1}}=\frac{x_{2}}{y_{2}}=\cdots=\frac{x_{n}}{y_{n}}$.

Proof. Using Hölder's inequality, we have

$$
\sum_{i=1}^{n} x_{i}=\sum_{i=1}^{n}\left(\frac{x_{i}}{\sqrt[q]{y_{i}}} \sqrt[q]{y_{i}}\right) \leq\left[\sum_{i=1}^{n}\left(\frac{x_{i}}{\sqrt[q]{y_{i}}}\right)^{p}\right]^{1 / p}\left[\sum_{i=1}^{n}\left(\sqrt[q]{y_{i}}\right)^{q}\right]^{1 / q}
$$

and

$$
\left[\sum_{i=1}^{n}\left(\sqrt[q]{y_{i}}\right)^{q}\right]^{1 / q}=\left(\sum_{i=1}^{n} \frac{x_{i}^{p}}{y_{i}^{p / q}}\right)^{1 / p}\left(\sum_{i=1}^{n} y_{i}\right)^{1 / q} .
$$

Combining the above inequality with the above equality yields (2.1). The proof of Lemma 2.1 is complete.

The integral form of Lemma 2.1 may be easily derived as follows. 
Lemma 2.2. Let $f(x)$ and $g(x)$ be positive and integrable functions on $[a, b]$. If $p>1$ and the functions $f^{p}(x)$ and $g^{p-1}(x)$ are integrable on $[a, b]$, then

$$
\int_{a}^{b} \frac{f^{p}(x)}{g^{p-1}(x)} \mathrm{d} x \geq \frac{\left[\int_{a}^{b} f(x) \mathrm{d} x\right]^{p}}{\left[\int_{a}^{b} g(x) \mathrm{d} x\right]^{p-1}}
$$

The equality in (2.2) holds if and only if $f(x)=k g(x)$.

Lemma 2.3. Let $f_{k}(x)$ and $g_{k}(x)$ for $k=1,2 \ldots, n$ be positive and integrable functions on $[a, b]$. If $1<p \leq 2$ and the functions $f_{k}^{p}(x)$ and $g_{k}^{p-1}(x)$ are integrable on $[a, b]$, then

$$
\sum_{k=1}^{n} \frac{\int_{a}^{b} f_{k}^{p}(x) \mathrm{d} x}{\int_{a}^{b} g_{k}^{p-1}(x) \mathrm{d} x} \geq \frac{\int_{a}^{b}\left[\sum_{k=1}^{n} f_{k}(x)\right]^{p} \mathrm{~d} x}{\int_{a}^{b}\left[\sum_{k=1}^{n} g_{k}(x)\right]^{p-1} \mathrm{~d} x} .
$$

Proof. We prove the inequality (2.3) by mathematical induction.

When $n=1$, the inequality (2.3) is trivial.

When $n=2$, the inequality (2.3) may be rewritten as

$$
\frac{\int_{a}^{b}\left[f_{1}(x)+f_{2}(x)\right]^{p} \mathrm{~d} x}{\int_{a}^{b}\left[g_{1}(x)+g_{2}(x)\right]^{p-1} \mathrm{~d} x} \leq \frac{\int_{a}^{b} f_{1}^{p}(x) \mathrm{d} x}{\int_{a}^{b} g_{1}^{p-1}(x) \mathrm{d} x}+\frac{\int_{a}^{b} f_{2}^{p}(x) \mathrm{d} x}{\int_{a}^{b} g_{2}^{p-1}(x) \mathrm{d} x} .
$$

Setting

$$
\begin{aligned}
& A_{1}=\left\|f_{1}(x)\right\|_{p}=\left[\int_{a}^{b} f_{1}^{p}(x) \mathrm{d} x\right]^{1 / p}, \\
& A_{2}=\left\|f_{2}(x)\right\|_{p}=\left[\int_{a}^{b} f_{2}^{p}(x) \mathrm{d} x\right]^{1 / p}, \\
& B_{1}=\left\|g_{1}(x)\right\|_{p-1}=\left[\int_{a}^{b} g_{1}^{p-1}(x) \mathrm{d} x\right]^{1 /(p-1)}, \\
& B_{2}=\left\|g_{2}(x)\right\|_{p-1}=\left[\int_{a}^{b} g_{2}^{p-1}(x) \mathrm{d} x\right]^{1 /(p-1)} .
\end{aligned}
$$

Then, the inequality (2.4) becomes

$$
\frac{A_{1}^{p}}{B_{1}^{p-1}}+\frac{A_{2}^{p}}{B_{2}^{p-1}} \geq \frac{\left\|f_{1}(x)+f_{2}(x)\right\|_{p}^{p}}{\left\|g_{1}(x)+g_{2}(x)\right\|_{p-1}^{p-1}} .
$$

By Lemma 2.2, we obtain

$$
\frac{A_{1}^{p}}{B_{1}^{p-1}}+\frac{A_{2}^{p}}{B_{2}^{p-1}} \geq \frac{\left(A_{1}+A_{2}\right)^{p}}{\left(B_{1}+B_{2}\right)^{p-1}} .
$$

So, in order to prove the inequality (2.5), it is sufficient to show

$$
\frac{\left[\left\|f_{1}(x)\right\|_{p}+\left\|f_{2}(x)\right\|_{p}\right]^{p}}{\left[\left\|g_{1}(x)\right\|_{p-1}+\left\|g_{2}(x)\right\|_{p-1}\right]^{p-1}} \geq \frac{\left\|f_{1}(x)+f_{2}(x)\right\|_{p}^{p}}{\left\|g_{1}(x)+g_{2}(x)\right\|_{p-1}^{p-1}} .
$$


Since $1<p \leq 2$, then $0<p-1 \leq 1$. Applying Minkowski's inequality leads to

$$
\left\|f_{1}(x)\right\|_{p}+\left\|f_{2}(x)\right\|_{p} \geq\left\|f_{1}(x)+f_{2}(x)\right\|_{p}
$$

and

$$
\left\|g_{1}(x)\right\|_{p-1}+\left\|g_{2}(x)\right\|_{p-1} \leq\left\|g_{1}(x)+g_{2}(x)\right\|_{p-1} .
$$

Considering the ratio between inequalities (2.8) and (2.9) results in (2.7).

Now assume that the inequality (2.3) is true for some $n=m \in \mathbb{N}$. Letting

$$
\left\{\begin{array} { l } 
{ p _ { i } ( x ) = f _ { i } ( x ) } \\
{ p _ { m } ( x ) = f _ { m } ( x ) + f _ { m + 1 } ( x ) }
\end{array} \text { and } \left\{\begin{array}{l}
q_{i}(x)=g_{i}(x) \\
q_{m}(x)=g_{m}(x)+g_{m+1}(x),
\end{array}\right.\right.
$$

where $i=1,2, \ldots, m-1$. By the inductive hypothesis and the inequality (2.4), we obtain

$$
\begin{aligned}
& \frac{\int_{a}^{b}\left[\sum_{k=1}^{m+1} f_{k}(x)\right]^{p} \mathrm{~d} x}{\int_{a}^{b}\left[\sum_{k=1}^{m+1} g_{k}(x)\right]^{p-1} \mathrm{~d} x}=\frac{\int_{a}^{b}\left[\sum_{k=1}^{m} p_{k}(x)\right]^{p} \mathrm{~d} x}{\int_{a}^{b}\left[\sum_{k=1}^{m} q_{k}(x)\right]^{p-1} \mathrm{~d} x} \leq \sum_{k=1}^{m} \frac{\int_{a}^{b} p_{k}^{p}(x) \mathrm{d} x}{\int_{a}^{b} q_{k}^{p-1}(x) \mathrm{d} x} \\
= & \sum_{k=1}^{m-1} \frac{\int_{a}^{b} f_{k}^{p}(x) \mathrm{d} x}{\int_{a}^{b} g_{k}^{p-1}(x) \mathrm{d} x}+\frac{\int_{a}^{b}\left[f_{m}(x)+f_{m+1}(x)\right]^{p} \mathrm{~d} x}{\int_{a}^{b}\left[g_{m}(x)+g_{m+1}(x)\right]^{p-1} \mathrm{~d} x} \leq \sum_{k=1}^{m+1} \frac{\int_{a}^{b} f_{k}^{p}(x) \mathrm{d} x}{\int_{a}^{b} g_{k}^{p-1}(x) \mathrm{d} x} .
\end{aligned}
$$

This means that the inequality (2.3) holds for $n=m+1$. Lemma 2.3 is thus proved inductively.

The discrete version of the inequality (2.3) in Lemma 2.3 may be stated as a corollary below.

Corollary 2.4. Let $x_{k, i}$ for $k=1,2, \ldots, m$ and $i=1,2, \ldots, n$ be positive numbers. If $1<p \leq 2$, then

$$
\sum_{i=1}^{n} \frac{\sum_{k=1}^{m} x_{k, i}^{p}}{\sum_{k=1}^{m} y_{k, i}^{p-1}} \geq \frac{\sum_{k=1}^{m}\left(\sum_{i=1}^{n} x_{k, i}\right)^{p}}{\sum_{k=1}^{m}\left(\sum_{i=1}^{n} y_{k, i}\right)^{p-1}} .
$$

Lemma 2.5. Let $a_{k}$ for $k=1,2, \ldots, n$ be nonnegative numbers. If $p \geq 1$, then

$$
\left(\sum_{k=1}^{n} a_{k}\right)^{p} \leq n^{p-1} \sum_{k=1}^{n} a_{k}^{p}
$$

Proof. This follows from the convexity of the function $f(x)=x^{p}$ for $p \geq 1$ and the well known Jensen's inequality.

\section{Main results}

Now we are in a position to generalize Theorems 1.1 to 1.3. 
Theorem 3.1. Let $f_{k}(x)$ for $k=1,2, \ldots, n$ be positive integrable functions on $[a, b]$. If $1<p \leq 2$ and

$$
\sum_{k=1}^{n} \int_{a}^{b} f_{k}(x) \mathrm{d} x \geq[n(b-a)]^{p-1},
$$

then

$$
\sum_{k=1}^{n} \int_{a}^{b} f_{k}^{p}(x) \mathrm{d} x \geq\left[\sum_{k=1}^{n} \int_{a}^{b} f_{k}(x) \mathrm{d} x\right]^{p-1} .
$$

Proof. Letting $g_{k}(x)=1$ in Lemma 2.3 results in

$$
\sum_{k=1}^{n} \frac{\int_{a}^{b} f_{k}^{p}(x) \mathrm{d} x}{\int_{a}^{b} 1^{p-1} \mathrm{~d} x} \geq \frac{\int_{a}^{b}\left[\sum_{k=1}^{n} f_{k}(x)\right]^{p} \mathrm{~d} x}{\int_{a}^{b}\left(\sum_{k=1}^{n} 1\right)^{p-1} \mathrm{~d} x} .
$$

Furthermore, by Lemma 2.2 and the condition (3.1), we have

$$
\begin{aligned}
\sum_{k=1}^{n} \int_{a}^{b} f_{k}^{p}(x) \mathrm{d} x & \geq \frac{1}{n^{p-1}} \int_{a}^{b}\left[\sum_{k=1}^{n} f_{k}(x)\right]^{p} \mathrm{~d} x=\frac{1}{n^{p-1}} \int_{a}^{b} \frac{\left[\sum_{k=1}^{n} f_{k}(x)\right]^{p}}{1^{p-1}} \mathrm{~d} x \\
& \geq \frac{1}{n^{p-1}} \frac{\left[\int_{a}^{b} \sum_{k=1}^{n} f_{k}(x) \mathrm{d} x\right]^{p}}{\left(\int_{a}^{b} 1 \mathrm{~d} x\right)^{p-1}} \geq\left[\int_{a}^{b} \sum_{k=1}^{n} f_{k}(x) \mathrm{d} x\right]^{p-1} .
\end{aligned}
$$

The proof is completed.

Remark 3.1. If $n=1$, Theorem 3.1 generalizes Theorem 1.1 in relatively strong condition.

Theorem 3.2. Let $f_{k}(x)$ for $k=1,2, \ldots, n$ be nonnegative continuous functions on $[a, b]$. If $\sum_{k=1}^{n} f_{k}(x)$ is increasing on $[a, b]$ and

$$
\sum_{k=1}^{n} f_{k}(x) \geq(p-1)(b-a)^{p-2} n^{p-1},
$$

then the inequality (3.2) holds true for all $p>1$.

Proof. For all $x \in[a, b]$, let

$$
H(x)=\sum_{k=1}^{n} \int_{a}^{x} f_{k}^{p}(t) \mathrm{d} t-\left[\int_{a}^{x} \sum_{k=1}^{n} f_{k}(t) \mathrm{d} t\right]^{p-1} .
$$

A simple computation yields

$$
H^{\prime}(x)=\sum_{k=1}^{n} f_{k}^{p}(x)-(p-1)\left[\int_{a}^{x} \sum_{k=1}^{n} f_{k}(t) \mathrm{d} t\right]^{p-2} \sum_{k=1}^{n} f_{k}(x) .
$$

Since $\sum_{k=1}^{n} f_{k}(x)$ is increasing on $[a, b]$, then

$$
0 \leq \int_{a}^{x} \sum_{k=1}^{n} f_{k}(t) \mathrm{d} t \leq(b-a) \sum_{k=1}^{n} f_{k}(x) .
$$


Further by Lemma 2.5, we have

$$
\begin{aligned}
H^{\prime}(x) & \geq \sum_{k=1}^{n} f_{k}^{p}(x)-(p-1)(b-a)^{p-2}\left[\sum_{k=1}^{n} f_{k}(x)\right]^{p-1} \\
& \geq \frac{1}{n^{p-1}}\left[\sum_{k=1}^{n} f_{k}(x)\right]^{p}-(p-1)(b-a)^{p-2}\left[\sum_{k=1}^{n} f_{k}(x)\right]^{p-1} \\
& =\left[\sum_{k=1}^{n} f_{k}(x)\right]^{p-1} \frac{\sum_{k=1}^{n} f_{k}(x)-(p-1) n^{p-1}(b-a)^{p-2}}{n^{p-1}} \\
& \geq 0 .
\end{aligned}
$$

Thus, the function $H(x)$ is increasing on $[a, b]$. In particular, $H(b) \geq H(a)=0$, which gives the desired inequality (3.2).

Theorem 3.3. Let $f_{k}(x)$ for $k=1,2, \ldots, n$ be nonnegative continuous on $[a, b]$ and differentiable on $(a, b)$, such that

$$
\sum_{k=1}^{n} f_{k}(a) \geq 0 \quad \text { and } \quad \sum_{k=1}^{n} f_{k}^{\prime}(x) \geq p
$$

Then we have

$$
\sum_{k=1}^{n} \int_{a}^{b} f_{k}^{p+2}(x) \mathrm{d} x \geq \frac{1}{n^{p+1}(b-a)^{p-1}}\left[\sum_{k=1}^{n} \int_{a}^{b} f_{k}(x) \mathrm{d} x\right]^{p+1}
$$

for all $p>1$.

Proof. Set

$$
G(x)=\sum_{k=1}^{n} \int_{a}^{x} f_{k}^{p+2}(t) \mathrm{d} t-\frac{1}{n^{p+1}(b-a)^{p-1}}\left[\int_{a}^{x} \sum_{k=1}^{n} f_{k}(t) \mathrm{d} t\right]^{p+1}
$$

for all $x \in[a, b]$. Simple computations and utilization of Lemma 2.5 and (3.4) yield

$$
\begin{aligned}
G^{\prime}(x) & =\sum_{k=1}^{n} f_{k}^{p+2}(x)-\frac{p+1}{n^{p+1}(b-a)^{p-1}}\left[\int_{a}^{x} \sum_{k=1}^{n} f_{k}(t) \mathrm{d} t\right]^{p} \sum_{k=1}^{n} f_{k}(x) \\
& \geq \frac{1}{n^{p+1}}\left[\sum_{k=1}^{n} f_{k}(x)\right]^{p+2}-\frac{p+1}{n^{p+1}(b-a)^{p-1}}\left[\int_{a}^{x} \sum_{k=1}^{n} f_{k}(t) \mathrm{d} t\right]^{p} \sum_{k=1}^{n} f_{k}(x) \\
& =\frac{\sum_{k=1}^{n} f_{k}(x)}{n^{p+1}} h(x)
\end{aligned}
$$

and

$$
\begin{aligned}
h^{\prime}(x)=(p+1)\left[\sum_{k=1}^{n} f_{k}(x)\right]^{p} \sum_{k=1}^{n} f_{k}^{\prime}(x)-\frac{(p+1) p}{(b-a)^{p-1}}\left[\int_{a}^{x} \sum_{k=1}^{n} f_{k}(t) \mathrm{d} t\right]^{p-1} \sum_{k=1}^{n} f_{k}(x) \\
\geq(p+1)\left[\sum_{k=1}^{n} f_{k}(x)\right]^{p}\left[\sum_{k=1}^{n} f_{k}^{\prime}(x)-p\right] \geq 0 .
\end{aligned}
$$

Since $h(x)$ is increasing on $[a, b]$ and $h(a)=\left[\sum_{k=1}^{n} f_{k}(a)\right]^{p+1} \geq 0$, the function $G(x)$ is also increasing on $[a, b]$. Especially, $G(b) \geq G(a)=0$, which gives the desired inequality (3.6). 
Remark 3.2. If $n=1$, Theorem 3.3 is just Theorem 1.3.

Corollary 3.4. Under conditions of Theorem 3.3 , when $[a, b]=[0,1]$, we have

$$
\sum_{k=1}^{n} \int_{0}^{1} f_{k}^{p+2}(x) \mathrm{d} x \geq \frac{1}{n^{p+1} p}\left[\sum_{k=1}^{n} \int_{0}^{1} f_{k}(x) \mathrm{d} x\right]^{p+1} .
$$

Proof. This follows from respectively replacing $f_{k}(x)$ by $p f_{k}(x)$ and $[a, b]$ by $[0,1]$ in Theorem 3.3.

Remark 3.3. If $n=1$, Corollary 3.4 becomes [3, p. 124, Corollary 3.1].

By the similar method as above, we may prove following Theorem 3.5.

Theorem 3.5. Let $f_{k}(x)$ for $k=1,2, \ldots, n$ be nonnegative and continuous on $[a, b]$ and be differentiable on $(a, b)$, such that

$$
\sum_{k=1}^{n} f_{k}(a) \geq 0 \quad \text { and } \quad \sum_{k=1}^{n} f_{k}^{\prime}(x) \geq \frac{2 n^{2 p}}{p+1} .
$$

Then

$$
\sum_{k=1}^{n} \int_{a}^{b} f_{k}^{2 p+1}(x) \mathrm{d} x \geq\left\{\int_{a}^{b}\left[\sum_{k=1}^{n} f_{k}(x)\right]^{p} \mathrm{~d} x\right\}^{2}
$$

for all $p>1$.

Remark 3.4. If $n=1$, Theorem 3.5 is the same as [3, p. 124, Proposition 1.1].

Theorem 3.6. Let $f_{k}(x)$ for $k=1,2, \ldots, n$ be nonnegative, continuous, and not identically zero on $[a, b]$ with $f_{k}(a)=0$, and let $\alpha, \beta$ be positive real numbers such that $\alpha>\beta>1$. If

$$
\left\{\left[\sum_{k=1}^{n} f_{k}(x)\right]^{(\alpha-\beta) /(\beta-1)}\right\}^{\prime} \geq \frac{(\alpha-\beta)\left(n^{\alpha-1} \beta\right)^{1 /(\beta-1)}}{\alpha-1}
$$

for all $x \in(a, b)$, then

$$
\sum_{k=1}^{n} \int_{a}^{b} f_{k}^{\alpha}(x) \mathrm{d} x \geq\left[\sum_{k=1}^{n} \int_{a}^{b} f_{k}(x) \mathrm{d} x\right]^{\beta} .
$$

Proof. Utilizing Lemma 2.5 and Cauchy's mean value theorem consecutively yields

$$
\begin{aligned}
\frac{\left[\int_{a}^{b} \sum_{k=1}^{n} f_{k}(x) \mathrm{d} x\right]^{\beta}}{\sum_{k=1}^{n} \int_{a}^{b} f_{k}^{\alpha}(x) \mathrm{d} x} & =\frac{\beta\left[\int_{a}^{\xi} \sum_{k=1}^{n} f_{k}(x) \mathrm{d} x\right]^{\beta-1} \sum_{k=1}^{n} f_{k}(\xi)}{\sum_{k=1}^{n} f_{k}^{\alpha}(\xi)} \\
& \leq \frac{\beta n^{\alpha-1}\left[\int_{a}^{\xi} \sum_{k=1}^{n} f_{k}(x) \mathrm{d} x\right]^{\beta-1}}{\left[\sum_{k=1}^{n} f_{k}(\xi)\right]^{\alpha-1}}
\end{aligned}
$$




$$
\begin{aligned}
& =\left\{\frac{\left(\beta n^{\alpha-1}\right)^{1 /(\beta-1)} \int_{a}^{\xi} \sum_{k=1}^{n} f_{k}(x) \mathrm{d} x}{\left[\sum_{k=1}^{n} f_{k}(\xi)\right]^{(\alpha-1) /(\beta-1)}}\right\}^{\beta-1} \\
& =\left\{\frac{\left(\beta n^{\alpha-1}\right)^{1 /(\beta-1)} \sum_{k=1}^{n} f_{k}(\theta)}{\frac{\alpha-1}{\beta-1}\left[\sum_{k=1}^{n} f_{k}(\theta)\right]^{\frac{\alpha-\beta}{\beta-1}} \sum_{k=1}^{n} f_{k}^{\prime}(\theta)}\right\}^{\beta-1} \\
& =\left\{\frac{\frac{\alpha-\beta}{\alpha-1}\left(\beta n^{\alpha-1}\right)^{1 /(\beta-1)}}{\left[\left(\sum_{k=1}^{n} f_{k}(\theta)\right)^{(\alpha-\beta) /(\beta-1)}\right]^{\prime}}\right\}^{\beta-1} \\
& \leq 1 .
\end{aligned}
$$

Thus, the inequality (3.11) follows.

Remark 3.5. If $n=1$, Theorem 3.6 is reduced to [32, p. 7, Theorem 1.1].

\section{Acknowledgements}

The authors thank the anonymous referees for their careful comments on and valuable suggestions to the original version of this manuscript.

\section{References}

[1] H. Agahi and M. A. Yaghoobi, A Feng Qi type inequality for Sugeno integral, Fuzzy Inf. Eng. 2 (2010), 293-304; Available online at http://dx.doi.org/10.1007/s12543-010-0051-8.

[2] M. Akkouchi, On an integral inequality of Feng Qi, Divulg. Mat. 13 (2005), no. 1, 11-19.

[3] M. Akkouchi, Some integral inequalities, Divulg. Mat. 11 (2003), no. 2, 121-125.

[4] L. Bougoffa, An integral inequality similar to Qi's inequality, J. Inequal. Pure Appl. Math. 6 (2005), no. 1, Art. 27; Available online at http: //www .emis . de/journals/JIPAM/article496.html.

[5] L. Bougoffa, Notes on Qi type integral inequalities, J. Inequal. Pure Appl. Math. 4 (2003), no. 4, Art. 77; Available online at http://www.emis.de/journals/JIPAM/article318.html.

[6] K. Brahim, N. Bettaibi, and M. Sellemi, On some Feng Qi type q-integral inequalities, J. Inequal. Pure Appl. Math. 9 (2008), no. 2, Art. 43; Available online at http: //www .emis.de/journals/JIPAM/article975.html.

[7] X.-K. Chai and H.-X. Du, Several discrete inequalities, Int. J. Math. Anal. 4 (2010), no. 33-36, 1645-1649.

[8] X.-K. Chai, Y.-G. Zhao, and H.-X. Du, Several answers to an open problem, Int. J. Contemp. Math. Sci. 5 (2010), no. 37, 1813-1817.

[9] Y. Chen and J. Kimball, Note on an open problem of Feng Qi, J. Inequal. Pure Appl. Math. 7 (2006), no. 1, Art. 4; Available online at http: //www .emis.de/journals/JIPAM/article621.html.

[10] V. Csiszár and T. F. Móri, The convexity method of proving moment-type inequalities, Statist. Probab. Lett. 66 (2004), no. 3, 303-313.

[11] Z. Dahmani, New inequalities of Qi type, J. Math. System Sci. 1 (2011), no. 1, 1-7.

[12] Z. Dahmani and N. Bedjaoui, Some generalized integral inequalities, J. Adv. Res. Appl. Math. 3 (2011), no. 4, 58-66.

[13] Z. Dahmani and S. Belarbi, Some inequalities of Qi type using fractional integration, Int. J Nonlinear Sci. 10 (2010), no. 4, 396-400. 
[14] Z. Dahmani and H. M. El Ard, Generalizations of some integral inequalities using Riemann-Liouville operator, Int. J. Open Probl. Comput. Sci. Math. 4 (2011), no. 4, 40-46.

[15] Z. Dahmani and L. Tabharit, Certain inequalities involving fractional integrals, J. Adv. Res. Sci. Comput. 2 (2010), no. 1, 55-60.

[16] Y. Hong, A note on Feng Qi type integral inequalities, Int. J. Math. Anal. (Ruse) 1 (2007), no. 25-28, 1243-1247.

[17] V. Krasniqi and A. Sh. Shabani, On some Feng Qi type h-integral inequalities, Int. J. Open Probl. Comput. Sci. Math. 2 (2009), no. 4, 516-521.

[18] W.-J. Liu, C.-C. Li, and J.-W. Dong, Consolidations of extended Qi's inequality and Bougoffa's inequality, J. Math. Inequal. 2 (2008), no. 1, 9-15.

[19] W.-J. Liu, C.-C. Li, and J.-W. Dong, Note on Qi's inequality and Bougoffa's inequality, J. Inequal. Pure Appl. Math. 7 (2006), no. 4, Art. 129; Available online at http://www.emis.de/journals/JIPAM/article746.html.

[20] W.-J. Liu, Q.-A. Ngô, and V. N. Huy, Several interesting integral inequalities, J. Math. Inequal. 3 (2009), no. 2, 201-212.

[21] S. Mazouzi and F. Qi, On an open problem regarding an integral inequality, J. Inequal. Pure Appl. Math. 4 (2003), no. 2, Art. 31; Available online at http://www . emis . de/journals/JIPAM/article269.html.

[22] Y. Miao, Further development of Qi-type integral inequality, J. Inequal. Pure Appl. Math. 7 (2006), no. 4, Art. 144; Available online at http://www.emis.de/journals/JIPAM/article763.html.

[23] Y. Miao and J.-F. Liu, Discrete results of Qi-type inequality, Bull. Korean Math. Soc. 46 (2009), no. 1, $125-134$.

[24] Y. Miao and F. Qi, Several q-integral inequalities, J. Math. Inequal. 3 (2009), no. 1, 115-121; Available online at http://dx.doi.org/10.7153/jmi-03-11.

[25] Q. A. Ngô and P. H. Tung, Notes on an open problem of $F$ Q $i$ and Y. Chen and J. Kimball, J. Inequal. Pure Appl. Math. 8 (2007), no. 2, Art. 41; Available online at http: //www .emis.de/journals/JIPAM/article856.html.

[26] J. Pečarić and T. Pejković, Note on Feng Qi’s integral inequality, J. Inequal. Pure Appl. Math. 5 (2004), no. 3 , Art. 51; Available online at http: //www .emis.de/journals/JIPAM/article418.html.

[27] J. Pečarić and T. Pejković, On an integral inequality, J. Inequal. Pure Appl. Math. 5 (2004), no. 2, Art. 47; Available online at http://www . emis .de/journals/JIPAM/article401.html.

[28] G. W. Peters, Y. Fan, and S. A. Sisson, On sequential Monte Carlo, partial rejection control and approximate Bayesian computation, Available online at http: //arxiv . org/abs/0808.3466.

[29] T. K. Pogány, On an open problem of F. Qi, J. Inequal. Pure Appl. Math. 3 (2002), no. 4, Art. 54; Available online at http://www.emis.de/journals/JIPAM/article206.html.

[30] F. Qi, Several integral inequalities, RGMIA Res. Rep. Coll. 2 (1999), no. 7, Art. 9, 1039-1042; Available online at http://rgmia.org/v2n7.php.

[31] F. Qi, Several integral inequalities, J. Inequal. Pure Appl. Math. 1 (2000), no. 2, Art. 19; Available online at http://www .emis.de/journals/JIPAM/article113.html.

[32] F. Qi, A.-J. Li, W.-Z. Zhao, D.-W. Niu, and J.-Cao, Extensions of several integral inequalities, J. Inequal. Pure Appl. Math. 7 (2006), no. 3, Art. 107; Available online at http: //www .emis.de/journals/JIPAM/article706.html.

[33] F. Qi and K.-W. Yu, Note on an integral inequality, J. Math. Anal. Approx. Theory 2 (2007), no. 1, 96-98.

[34] S. Saitoh, V. K. Tuan, and M. Yamamoto, Reverse convolution inequalities and applications to inverse heat source problems, J. Inequal. Pure. Appl. Math. 3 (2002), no. 5, Art. 80; Available online at http://www.emis.de/journals/JIPAM/article232.html.

[35] M. Z. Sarikaya, U. M. Ozkan, and H. Yildirim, Time scale integral inequalities similar to Qi's inequality, J. Inequal. Pure Appl. Math. 7 (2006), no. 4, Art. 128; Available online at http: //www . emis.de/journals/JIPAM/article745.html.

[36] M. R. Segi Rahmat, On some $(q, h)$-analogues of integral inequalities on discrete time scales, Comput. Math. Appl. 62 (2011), no. 4, 1790-1797; Available online at http://dx.doi.org/10.1016/j.camwa.2011.06.022. 
[37] W. T. Sulaiman, A study on new q-integral inequalities, Appl. Math. 2 (2011), no. 4, 465-469; Available online at http://dx.doi.org/10.4236/am.2011.24059.

[38] W. T. Sulaiman, Several q-integral inequalities, Aust. J. Math. Anal. Appl. 7 (2010), no. 2, Art. 6; Available online at http: //ajmaa. org/cgi-bin/paper.pl?string=v7n2/V7I2P6.tex.

[39] J.-S. Sun, A note on an open problem for integral inequality, RGMIA Res. Rep. Coll. 7 (2004), no. 3, Art. 21, 539-542; Available online at http://rgmia.org/v7n3.php.

[40] J.-S. Sun and Y.-Z. Wu, Note on an open problem of inequality, College Math. (Dàxué Shùxué) 24 (2008), no. 1, $126-128$.

[41] N. Towghi, Notes on integral inequalities, RGMIA Res. Rep. Coll. 4 (2001), no. 2, Art. 12, 277-278; Available online at http://rgmia.org/v4n2.php.

[42] A. Witkowski, On a F. Qi integral inequality, J. Inequal. Pure Appl. Math. 6 (2005), no. 2, Art. 36; Available online at http://www .emis .de/journals/JIPAM/article505.html.

[43] B.-Y. Xi, Some new inequalities of Qi type for definite integrals, The Scientific World Journal-Mathematical Analysis 2013 (2013), in press.

[44] P. Yan and M. Gyllenberg, On an open problem of integral inequalities, J. Inequal. Pure Appl. Math. 7 (2006), no. 5, Art. 170; Available online at http: //www . emis . de/journals/JIPAM/article788.html.

[45] P. Yan and M. Gyllenberg, On a conjecture of Qi-type integral inequalities, J. Inequal. Pure. Appl. Math. 7 (2006), no. 4, Art. 146; Available online at http://www . emis . de/journals/JIPAM/article760.html.

[46] L. Yin, On several new Qi’s inequalities, Creat. Math. Inform. 20 (2011), no. 1, 90-95.

[47] L. Yin, Q.-M. Luo, and F. Qi, Several integral inequalities on time scales, J. Math. Inequal. 6 (2012), no. 3, 419-429; Available online at http://dx.doi .org/10.7153/jmi-06-39.

[48] K.-W. Yu and F. Qi, A short note on an integral inequality, RGMIA Res. Rep. Coll. 4 (2001), no. 1, Art. 4, 23-25; Available online at http://rgmia.org/v4n1.php.

Department of Mathematics, Binzhou University, Binzhou City, Shandong Province, 256603, China.

E-mail: yinli_79@163.com

College of Information and Business, Zhongyuan University of Technology, Zhengzhou City, Henan Province, 450007, China.

E-mail: nnddww@gmail.com

Department of Mathematics, College of Science, Tianjin Polytechnic University, Tianjin City, 300387, China.

E-mail: qifeng618@gmail.com 